This item was submitted to Loughborough's Research Repository by the author.

Items in Figshare are protected by copyright, with all rights reserved, unless otherwise indicated.

\title{
Location dependent transaction for mobile environment
}

PLEASE CITE THE PUBLISHED VERSION

PUBLISHER

(C) IEEE

VERSION

VoR (Version of Record)

LICENCE

CC BY-NC-ND 4.0

REPOSITORY RECORD

Al-Qerem, Ahmad H., and Walter Hussak. 2019. "Location Dependent Transaction for Mobile Environment". figshare. https://hdl.handle.net/2134/4166. 
This item was submitted to Loughborough's Institutional Repository (https://dspace.lboro.ac.uk/) by the author and is made available under the following Creative Commons Licence conditions.

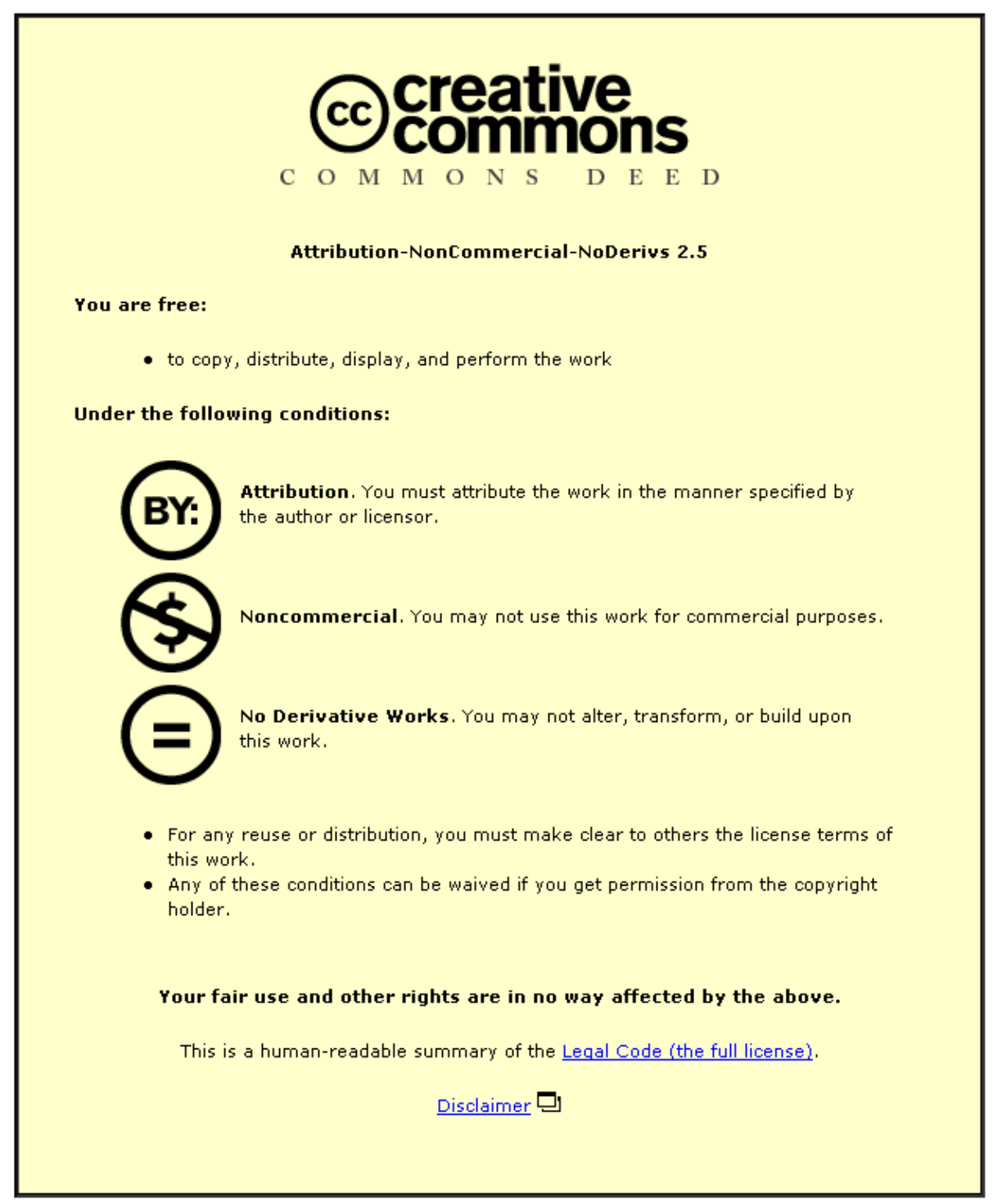

For the full text of this licence, please go to: http://creativecommons.org/licenses/by-nc-nd/2.5/ 


\title{
Location Dependent Transaction for Mobile Environment
}

\author{
Ahmad Alqerem (1), Walter Hussak (2) \\ (1) Computer science, Zarka Private University \\ B.o.Box 2000 -Zarka 13110, Zarka, Jordan \\ +962(0)795026670,+962(5)3821120 ahmad_qerm@zpu.edu.jo \\ (2) Computer science department, Loughborough University \\ Loughborough University Leicestershire LE11 UK \\ +44(0)1509 263171,+44(0) 1509 223909, w.hussak@lboro.ac.uk
}

\begin{abstract}
With recent advances of mobile and portable devices, more than one billion cellular phones in the world joined by other wireless handheld computing devices like personal digital assistants (PDAs) or pocket PCs, with this number of users there are significant opportunities for mobile commerce growth. Although mobile commerce enables access to goods and service regardless of the location of either buyer or seller, in many situations the specific location of the buyer and seller is critical to the transaction [1]. Also the time for transaction execution become increasingly important not from performance point view but also from the corresponding relationship between the data and location especially when the mobile user change its location dynamically.

In this paper we aim to introduce a mobile transaction model that takes into consideration the location dependent transaction and the time constraint for mobile transaction execution.
\end{abstract}

\section{Introduction}

In this paper we proposed a frame work for a location dependent mobile transaction that allow the mobile transaction to access a multi database and change its location during their execution and this framework is compatible with UMTS all -IP Network and use the same relocation schema [2]. The system architecture of our model is shown in the figure 1 , each level contain the data abstraction (Meta data) of the below level. The paper is organized as follow, section2 introduce the formal model for location dependent mobile transaction in section 3 we give some clarifying example in section 3 we introduce the concurrency control $t$ schema for managing multiple location dependent transaction ,section 4 conclude our work

\section{Formal model}

Location dependent mobile transaction is defined by a 3tuple ( $\mathrm{S}, \mathrm{D}, \mathrm{P})$ where $\mathrm{S}$ is a set of sub- transaction and $D$ represent the set of dependencies that define over a power set of $\mathrm{S}$ and $\mathrm{P}$ is a set of predicate (T,L,Q) associated with each subtransaction, where $T$ is the deadline for location dependent subtransaction execution and this will not be exceeded and $L$ which represent the type of each subtransaction if its location dependent or not, Q which represent any other condition to be satisfied before transaction execution . an optimistic concurrency control technique based on the timestamp ordering are used to deal with different concurrent subtransactions from different location dependent mobile transaction that investigate the set of predicate $P$, in the scheduling protocol. a simulation study have been conducted and show how this model effectively deal with a mobile transaction which access multidatabase system while moving from one location to another.

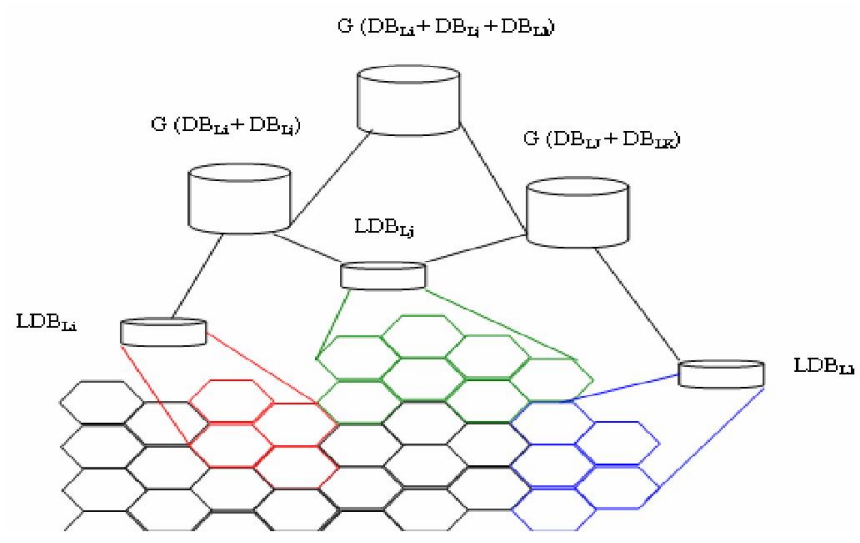

Figure1

The set of dependency D can represent any sort of transaction model starting from flat transaction which constitute of a set of read write operation with a partially ordered relationship (i.e. there is one subtransaction and D is empty ) to the advanced transactions model depend on the semantic of the application which used for.

Q may used to help increasing concurrency (i.e. there are tow subtransaction from different mobile transaction tij, tkl and tij need a data items $S$ and tkl need a data items $\mathrm{U}$ and $\mathrm{S}$ is contained in $\mathrm{U}$, or the region where $\mathrm{S}$ is a 
valid data is cover the region where the $U$ is valid, or the time interval for the tij is contained in the time interval needed to accomplish tkl)

Each location govern by a manager which is responsible with other locations managers to communicate with the GTM of the database to execute and commit the transactions who cross over more than one location

\section{Example:}

The user $\mathrm{X}$ is looking for booking a restaurant at 2:00 $\mathrm{pm}$ at lunch time with budget $10 \$$ and after that he want to go to cinema to see a thrill movie at $4: 00 \mathrm{pm}$ also he have a condition that the restaurant and the cinema must have a park and the distance between the restaurant and the cinema not exceed $2 \mathrm{~km}$ and this will start at the time of issuing the transaction then he specify that the confirmation of booking these facilities must be within 5 $\mathrm{km}$ of his current location and not exceed 1:00 pm .

T1: booking restaurant

T2: booking cinema

T3: check park availability

T4: check credit card availability

$\mathrm{S}=\{\mathrm{t} 1, \mathrm{t} 2, \mathrm{t} 3, \mathrm{t} 4\}$

$\mathrm{D}=\{\mathrm{t} 4-\mathrm{t} 3-\mathrm{t} 1, \mathrm{t} 4, \mathrm{t} 3, \mathrm{t} 2\},\{\mathrm{R} 1-\mathrm{Cl}<2 \mathrm{~km}\}$ where $\mathrm{RL}$, $\mathrm{CL}$ are a restaurant location and cinema location respectively

$\mathrm{P} 1=(2: 00 \mathrm{pm}, 5 \mathrm{~km}, 10 \$)$

$\mathrm{P} 2=(4: 00 \mathrm{pm}, 5 \mathrm{~km}$, thrill movie $)$

Now depend on the user current location and the meta data with their location and time condition specifies by the transaction ,these data have to be send to the most generic node that meet all the requirement for this transaction starting from the closet node to the user current location and then further to the upper generic node

The concurrency control algorithm based on time stamp ordering are introduce to solve the scheduling problem arise from this complicated situation[3], we assume here that the global data base represent more than one agency for each special type of facilities (i.e. the restaurant agency contain all information about all the restaurant in the location covered by this agency)

So the problem arise when there is more than one location dependent transaction looking for the same facilities and the location specified by their transactions are overlapped in term of time and region, so the algorithm must be solve the conflict based on criteria specified in the predicate for each subtransaction, in addition to that we need to investigate the intra dependencies between the subtransaction that constitute the transaction to increase the concurrency and ensure the global serializability for all transactions

\section{Composite Timestamp Ordering Algorithm}

The Composite Timestamp Ordering CTO imposes an a priori total order on all multi location transactions [3] The CTO algorithm assigns and validates timestamp orders as follows all delegated subtransactions (the subtransaction that send to database component under the same multi database agency) are tagged as multi location and inherit the global timestamp from their parent transaction

Untagged transactions that enter a location multidatabase are new transactions submitted directly from an application New transactions are first decomposed If any of the resulting subtransactions are delegated to other multidatabase location then both they and their parent are tagged as multi locations and assigned the same unique timestamp Tagged transactions are delegated from other multidatabase location These transactions are first validated by checking that their timestamp is not older than that of the last committed transaction Tlast If it is older it is aborted_Otherwise it is decomposed All resulting subtransactions inherit the tags and the unique timestamp from the parent and are submitted to component databases for execution

The CTO algorithm ensures that new and preparing delegated subtransactions execute and commit in timestamp order The global concurrency control scheduler of each location maintains a multi location commit order list MLOL The MLOL is never empty because it always maintains Tlast as a node Every multi location transaction is added to the MLOL sorted on their timestamp value and placed in a running state When any subtransaction on the list is ready to prepare it first checks if it is older than Tlast If it is older it is aborted Otherwise it checks if any younger transactions are prepared If so it is placed in a waiting state and rechecks after a set interval or after a prepared transaction is forced to abort through the $2 \mathrm{PC}$ protocol Once a transaction passes the validation step it prepares enters a prepare state and waits for a commit signal from its $2 \mathrm{PC}$ coordinator On receiving a commit a prepared transaction aborts all older transactions in the running and waiting states then commits If it receives an abort signal it is removed from MLOL and signals all older transactions in the waiting state 


\section{Conclusion}

The significant issues such as location transparency scalability performance and

administration make multidatabase location composition inevitable We have shown that scheduling transactions that span multiple multidatabase location with predefined predicate associated with each subtransaction in term of time and location imply a matter of guaranteeing transaction atomicity and serializability at each multidatabase location. To guarantee global serializability in a composite multidatabase environment ordering constraints must be imposed on multi location transactions. We presented a general transaction model that may fit for any type of application in the mobile environment and proposed a transaction scheduling algorithms based on the time stamp ordering that ensure the serializability of more than one location dependent transaction accessing the same common multi databases based on their location.

\section{References}

[1] Defining A. Elmagarmid, $Y$. Leu, and $M$. Rusinkiewics. A Multidatabase Transaction Model for INTERBASE. In International Conference on $V L D B$, August 1990.

[2] Anna Ha'c, Senior Member, IEEE, and Bo Liu ; Database and Location Management Schemes for Mobile Communications;IEEE/ACM TRANSACTIONS ON NETWORKING, VOL. 6, NO. 6, DECEMBER 1998

[3] Veijalainen, Jari \& Terziyan, Vagan. Transaction Management for M-Commerce at a Mobile Terminal. 20th IEEE Symposium on Reliable Distributed Systems, Workshop on Reliable and Secure Applications in Mobile Environment, New Orleans, USA, October 28$31,2001$. 